\title{
Systematic review of traditional Chinese medicine Lianhua Qingwen in the treatment of new coronavirus pneumonia
}

\author{
Bo $\mathrm{Li}^{1}$ and Qing $\operatorname{Ren}^{2 *}$ \\ ${ }^{1}$ Department of Operation Room, The Third Xiangya Hospital of Central South University, China \\ ${ }^{2}$ Department of Orthopaedics and Traumatology, Li Ka Shing Faculty of Medicine, The University of Hong Kong, Hong Kong SAR, China
}

\begin{abstract}
Objective To systematically evaluate the effectiveness of traditional Chinese medicine Lianhua Qingwen in the treatment of novel Coronavirus Pneumonia (NCP). Methods The databases of PubMed, Embase, Cochrane Central, CBM, CNKI, Wanfang and VIP were searched by computer. The clinical studies of Lianhua Qingwen combined with conventional medicine for NCP were collected. The search time was established from each database to February 2020. Meta analysis was performed using Stata 12.0 software. Results Three trials were finally included in a total of 138 patients. Meta analysis results showed that Lianhuaqingwen combined with conventional drugs had a better disappearance rate of main symptoms (fever, cough, fatigue) and other symptoms (chest tightness, dyspnea, and loss of appetite) of NCP than conventional drugs. Conclusion The results of this systematic review show that, compared with conventional drugs, the Lianhuaqingwen combined group can better alleviate the main symptoms of patients, and at the same time, the symptoms of dyspnea and loss of appetite can be relieved. However, due to the low quality of the included literature, a high-quality, multi-center, large-sample, randomized, double-blind, controlled trial is needed to verify.
\end{abstract}

\section{Introduction}

Novel Coronavirus Pneumonia (NCP) is pneumonia infected with the new Coronavirus (2019-nCoV). The main symptoms are fever, cough, fatigue, and dyspnea. Severe and critically ill patients may develop respiratory failure and renal function. Failure is lifethreatening [1], and the NCP mortality rate is reported to be about $4.3 \%$ [2]. The main route of transmission is droplet transmission and contact transmission. It has the characteristics of strong infectivity, fast dissemination speed and general infection of the population, and the incubation period is 1-24 days [3]. For NCP currently without specific treatment drugs, mainly to maintain water and electrolyte balance, maintain internal environment stability, oxygen therapy, antiviral therapy, antibacterial therapy, hormone therapy, etc. The integration of traditional Chinese and western medicine played an important role in the prevention and control of the SARS epidemic in 2003. Since the NCP outbreak, many patients have received the treatment of the Chinese medicine lotus blast and have achieved good results.

Traditional Chinese medicine Lianhua Qingwen contains forsythia, honeysuckle, ephedra, fried almonds, gypsum, isatis root, Mianma Guanzhong, houttuynia cordata, patchouli, rhubarb, rhodiola, menthol, etc. [4] Broad-spectrum antiviral, anti-inflammatory and bacteriostatic effects. Some studies have suggested that Lianhua Qingwen combined with conventional drug treatment can significantly improve the symptoms of fever, cough, fatigue, and dyspnea in patients. Therefore, we conducted a systematic review to analyze the efficacy of Lianhua Qingwen combined with conventional drugs in the treatment of NCP.

\section{Materials and methods}

\section{Inclusion criteria}

Research Type: A clinical study to evaluate the efficacy of Lianhua Qingwen combined with conventional drugs in the treatment of NCP.

\section{Research objects:}

(1) Inclusion criteria:

Meets the diagnostic criteria of NCP and is a general type of inpatient.

\section{(2) Literature exclusion criteria:}

(1) Patients with severe and critical NCP; (2) Acute respiratory diseases not caused by 2019-n CoV; (3) Any other chronic respiratory diseases, respiratory bacterial infections such as purulent tonsillitis, acute tracheobronchitis, sinusitis, otitis media and other effects Respiratory diseases evaluated by clinical trials; (4) Basic diseases such as severe primary immunodeficiency disease, acquired immunodeficiency syndrome, congenital respiratory malformation, congenital heart disease, and abnormal lung development

Interventions: Test group: Lianhua Qingwen combined with conventional drugs; control group: conventional drug group.

Outcome indicators: Main indicators: disappearance rate of fever, cough, and fatigue.

Secondary indicators: disappearance of chest tightness, dyspnea, and loss of appetite.

${ }^{\star}$ Correspondence to: Qing Ren, Department of Orthopaedics and Traumatology, Li Ka Shing Faculty of Medicine, The University of Hong Kong, Hong Kong SAR, China, E-mail: renqing@hku.hk

Key words: pleurotus glutinosa, NCP, systematic review, randomized controlled trial Received: March 16, 2020; Accepted: March 24, 2020; Published: March 27, 2020 


\section{Retrieval strategy}

Search the PubMed, Embase, Cochrane Central, CBM, CNKI, Wanfang and VIP databases. The search period is from the establishment of the database to February 2020. Chinese search strategy: ("Lianhua Qingwen Capsule" RR "Lianhua Qingwen Granule” AND (“New Coronavirus Pneumonia" RR "New Coronary Pneumonia” RR "New Coronavirus Infected Pneumonia"). Traditional Chinese Medicine Lianhua Qingwen "RR" Lianhua Qingwen Granule "RR" Lianhua Qingwen") AND ("Novel Coronavirus Pneumonia "RR” NCP”) RR ("Novel Coronavirus-infected Pneumonia") RR (“NCIP”) RR (“2019 -nCoV”) RR (“COVID-19") RR (“SARS-CoV-2"). Search for gray literature, regardless of language.

\section{Data collection and analysis}

Data extraction and management: The two researchers independently extracted information. The data extraction table includes: (1) general information: title, author, date of publication, etc.; (2) research characteristics: document design and quality, specific content and implementation methods of research measures, and main tests. Results and so on; (3) Results measurement: disappearance rate of symptoms and signs.

Statistical methods: Meta analysis was performed using Stata 12.0 software. Heterogeneity between the results of the included studies was tested by $\mathrm{Q}$. When there is statistical homogeneity between the results of each study $(\mathrm{P}>0.1, \mathrm{I}[2]<50 \%)$, a fixed effect model is used for analysis; if there is statistical heterogeneity between the results of each study $(\mathrm{P}<$ $0.1, \mathrm{I}[2]>50 \%)$, when there is statistical heterogeneity between the two study groups without clinical heterogeneity, the random effect model is used for analysis; otherwise, only descriptive analysis is performed. Funnel chart analysis was used to detect publication bias.

\section{Results}

\section{Characteristics of included clinical trials}

Literature search results: According to the established search strategy, 3 related literatures were found, and a total of 138 patients were retrieved. The literature search process is shown in Figure 1.

Basic information of included studies: One of the included studies was a randomized controlled trial, one was a self-contrast control,

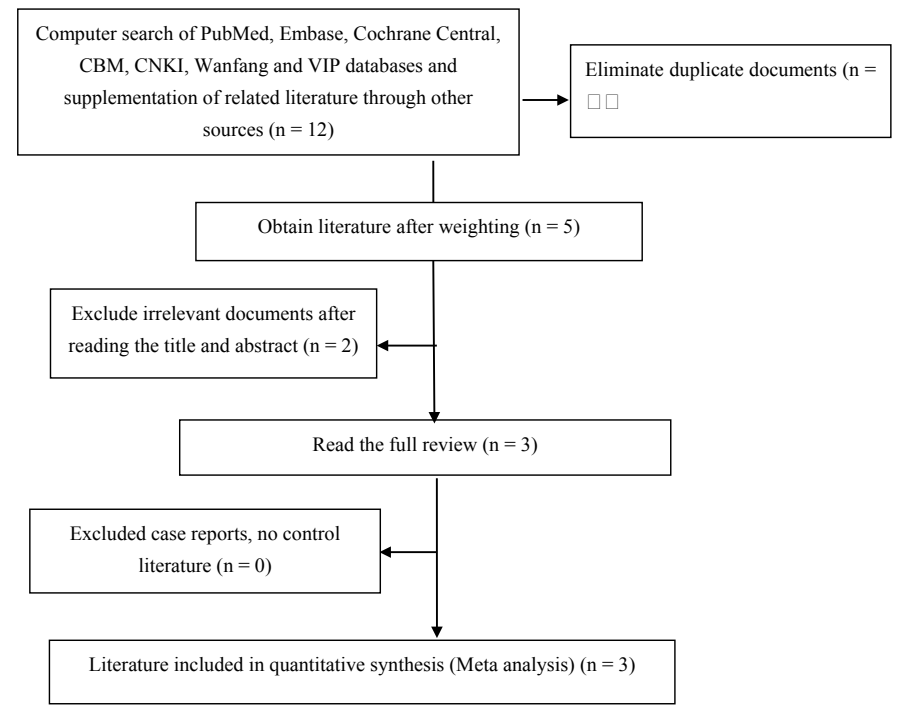

Figure 1. Document search and inclusion strategy flowchart and one was a retrospective control; the included population were all adults; 3 studies [5-7] applied western medicine diagnostic criteria, all studies had no withdrawal reports and ITT analysis; only 2 studies [5,7] mentioned adverse reactions; 3 studies were not followed up. The main characteristics of the included studies are shown in Table 1.

\section{Meta analysis results}

Fever disappearance: Three literatures [5-7] compared the disappearance rate of fever in NCP patients with Lianhua Qingwen combined with conventional drugs and conventional drugs. There was statistical heterogeneity among the included study groups $(\mathrm{P}=0.003$, I $[2]=82.8 \%$ ), and a random effects model was used for analysis. The results showed that the fever disappearance rate of Lianhuaqingwen combined with conventional drugs was higher than that of conventional drugs $[\mathrm{RR}=1.76,95 \% \mathrm{CI}(1.05,2.96), \mathrm{P}=0.033]$ (Figure 2).

Cough disappeared: Three literatures [5-7] compared the disappearance of cough in patients with NCP with Lianhua Qingwen combined with conventional drugs and conventional drugs. There was no statistical heterogeneity between the results in the control group $(\mathrm{P}=$ 0.268 , I [2] $=24.0 \%)$. The results showed that the cough disappearance rate of Lianhuaqingwen combined with conventional medicine was higher than that of conventional medicine group $[\mathrm{RR}=1.96,95 \% \mathrm{CI}$ $(1.43,2.68), \mathrm{P}<0.000$ ] (Figure 3).

Disappearance of fatigue: Three literatures [5-7] compared the disappearance rate of fatigue of NCP patients with Lianhua Qingwen combined with conventional drugs and conventional drugs. There was no statistical heterogeneity among the included study groups $(\mathrm{P}=0.14$, I [2] $=49.2 \%)$. The rate of fatigue loss of Lianhuaqingwen combined with conventional medicine was higher than that of conventional medicine group $[\mathrm{RR}=1.77,95 \% \mathrm{CI}(1.36,2.30), \mathrm{P}<0.0000]$ (Figure 4).

Disappearance of chest tightness: Three literatures [5-7] compared the disappearance rate of chest tightness in NCP patients with Lianhua Qingwen combined with conventional drugs and conventional drugs. There was statistical heterogeneity among the included study groups $(\mathrm{P}=0.003, \mathrm{I}[2]=82.8 \%)$. The disappearance rate of chest tightness of Lianhuaqingwen combined with conventional medicine was higher than that of conventional medicine group $[\mathrm{RR}=2.19,95 \% \mathrm{CI}(0.89$, 5.40), $\mathrm{P}=0.088$ ] (Figure 5).

Disappeared dyspnea: Three literatures [5-7] compared the disappearance rate of dyspnea in NCP patients with Lianhua Qingwen combined with conventional drugs and conventional drugs. There was no statistical heterogeneity among the included study groups $(\mathrm{P}=0.212$, I [2] $=35.5 \%)$. The dyspnea disappearance rate of Lianhuaqingwen combined with conventional medicine was higher than that of conventional medicine group $[\mathrm{RR}=4.58,95 \% \mathrm{CI}(2.39$, 8.79), $\mathrm{P}<0.0000$ ] (Figure 6).

Loss of appetite: Three literatures [8] compared the disappearance rates of Lianhuaqingwen combined with conventional drugs and conventional drugs in the loss of appetite in NCP patients. There was no statistical heterogeneity among the included study groups $(\mathrm{P}=0.361$, I $[2]=1.9 \%)$. The dyspnea disappearance rate of Lianhuaqingwen combined with conventional medicine was higher than that of conventional medicine group $[\mathrm{RR}=1.36,95 \% \mathrm{CI}(1.00,1.84), \mathrm{P}<$ 0.048] (Figure 7).

Incidence of adverse reactions: Two literatures compared the incidence of adverse reactions to COVID-19 with Lianhua Qingwen combined with conventional drugs and conventional drugs, and found no difference. 
Table 1. Main characteristics of the 3 studies that were finally included in the meta-analysis

\begin{tabular}{|c|c|c|c|c|c|c|c|c|c|c|}
\hline $\begin{array}{l}\text { Included } \\
\text { studies }\end{array}$ & $\begin{array}{l}\text { issuing } \\
\text { time }\end{array}$ & country & Type of Study & $\begin{array}{l}\text { diagnosis } \\
\text { method }\end{array}$ & $\begin{array}{c}\text { Experimental group } \\
\text { (number) (male / female) }\end{array}$ & $\begin{array}{l}\text { Control group } \\
\text { (number) }\end{array}$ & $\begin{array}{l}\text { Study } \\
\text { period }\end{array}$ & $\begin{array}{c}\text { Fever } \\
\text { (number) }\end{array}$ & $\begin{array}{c}\text { Cough } \\
\text { (number) }\end{array}$ & $\begin{array}{c}\text { Fatigue } \\
\text { (number) }\end{array}$ \\
\hline $\begin{array}{c}\text { Cheng } \\
\text { Dezhong [7] }\end{array}$ & $2020-2-19$ & China & $\begin{array}{l}\text { Prospective } \\
\text { study }\end{array}$ & $\begin{array}{l}\text { Positive nucleic } \\
\text { acid test }\end{array}$ & $54(29 / 25)$ & $54^{*}$ & 30日 & 40 & 30 & 54 \\
\hline Yao Kaitao [6] & $2020-2-6$ & China & $\begin{array}{l}\text { Prospective } \\
\text { study }\end{array}$ & $\begin{array}{l}\text { Positive nucleic } \\
\text { acid test }\end{array}$ & $21(16 / 5)$ & $21(12 / 9)$ & 19日 & 21 & 15 & 12 \\
\hline Lu Ruibing [5] & $2020-2-17$ & China & $\begin{array}{l}\text { Prospective } \\
\text { study }\end{array}$ & $\begin{array}{l}\text { Positive nucleic } \\
\text { acid test }\end{array}$ & $63(28 / 35)$ & $38(18 / 20)$ & 26日 & 60 & 54 & 40 \\
\hline
\end{tabular}

* Self-contrast

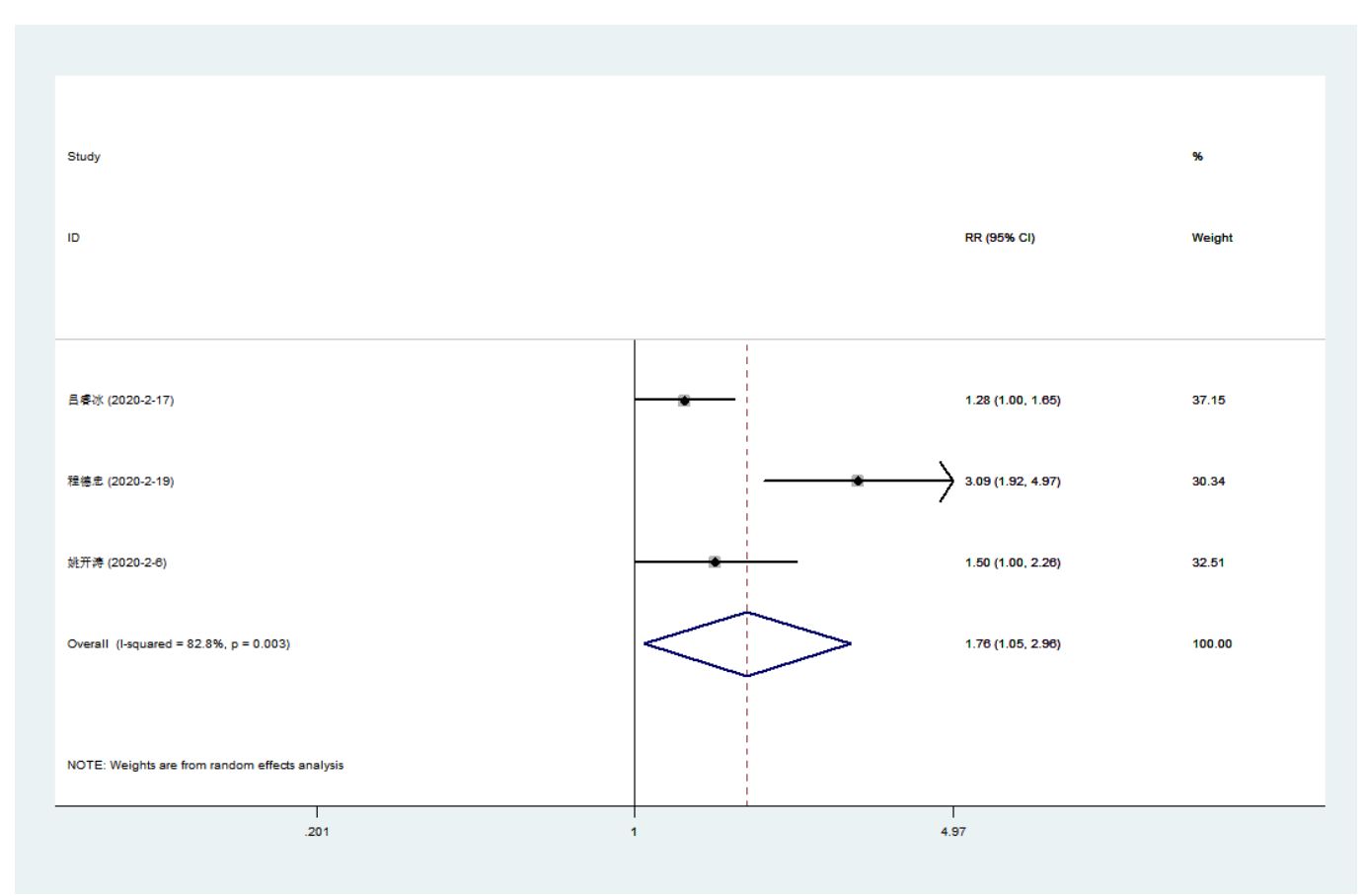

Figure 2. Meta-analysis forest plot of fever disappearance between the combined group and the control group

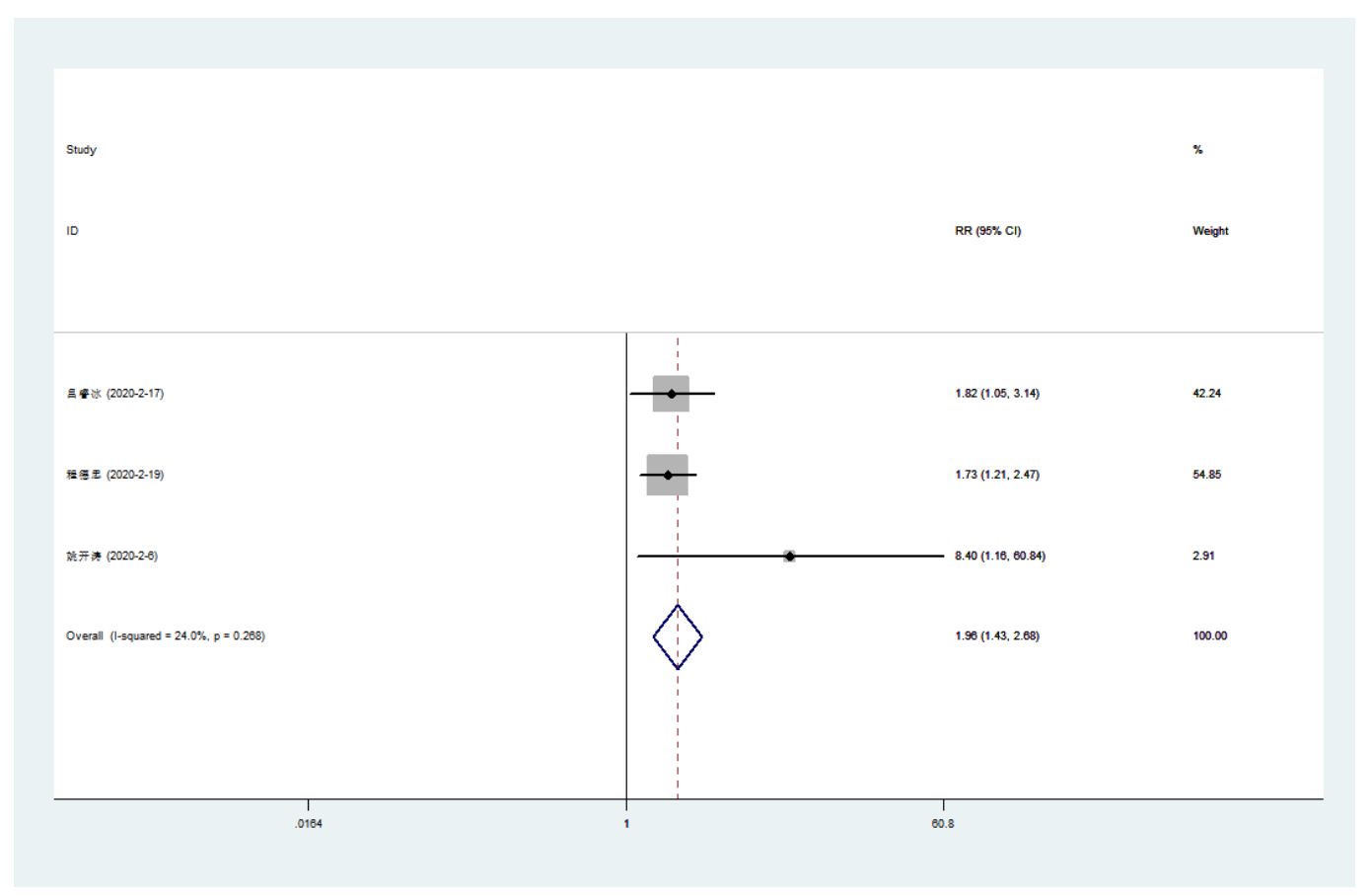

Figure 3. Meta-analysis forest plot of cough disappearance between the combined group and the control group 


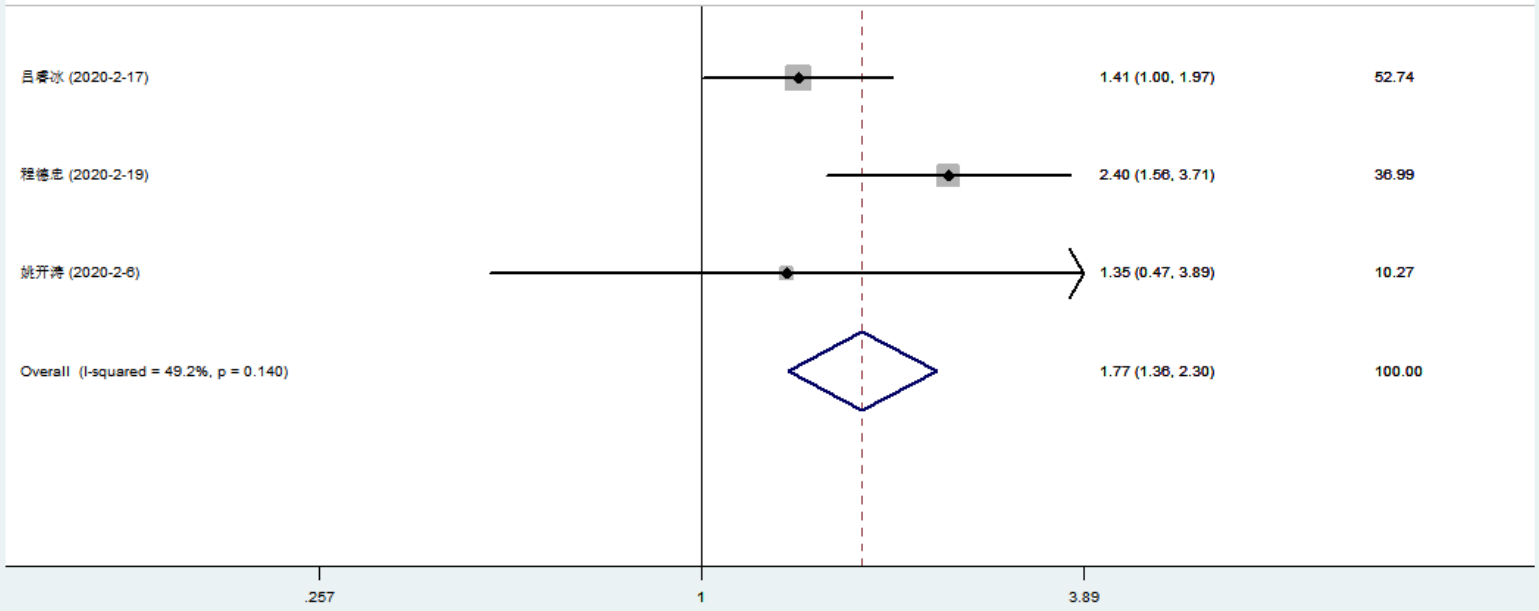

Figure 4. Meta-analysis forest plot of the disappearance of fatigue between the combined group and the control group

Study

ID

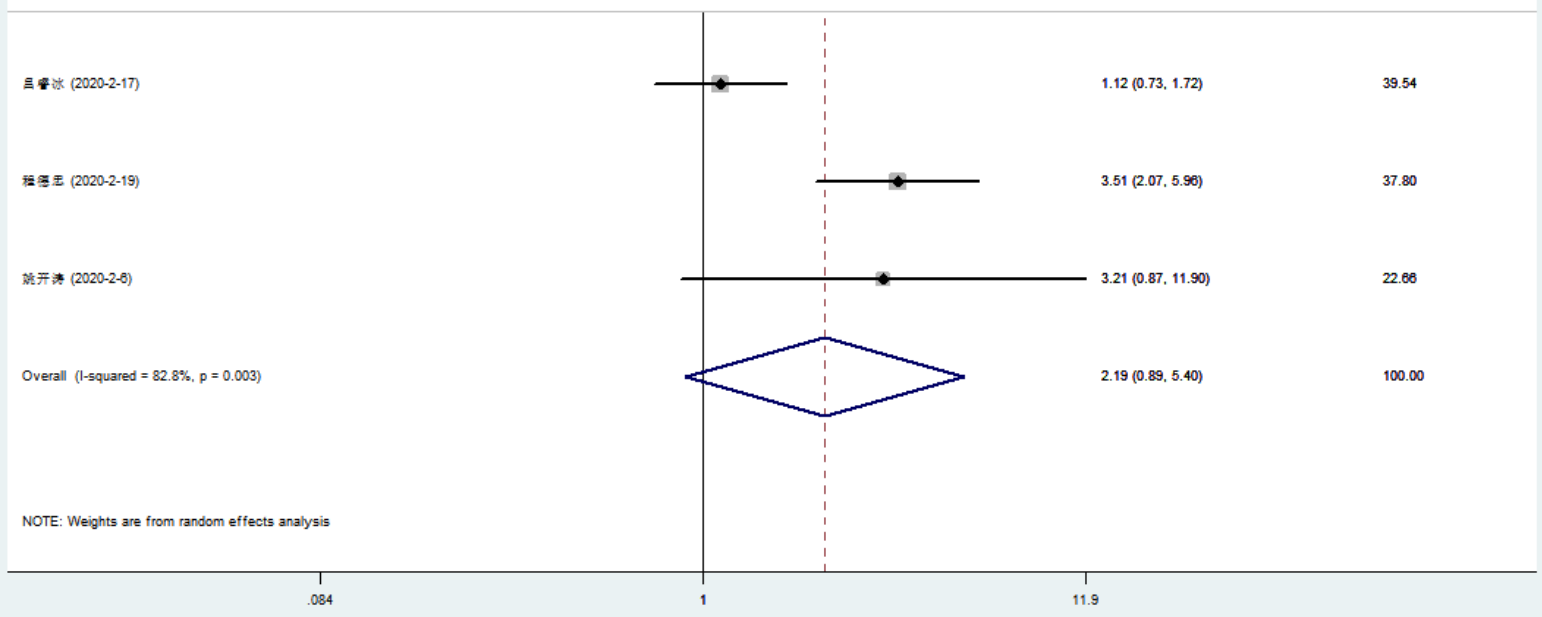

Figure 5. Meta-analysis forest plot of the disappearance of chest tightness between the combined group and the control group 
Study

10

路开: $(2020-2-8)$

Overall (1-squared $=35.5 \%, p=0.212$
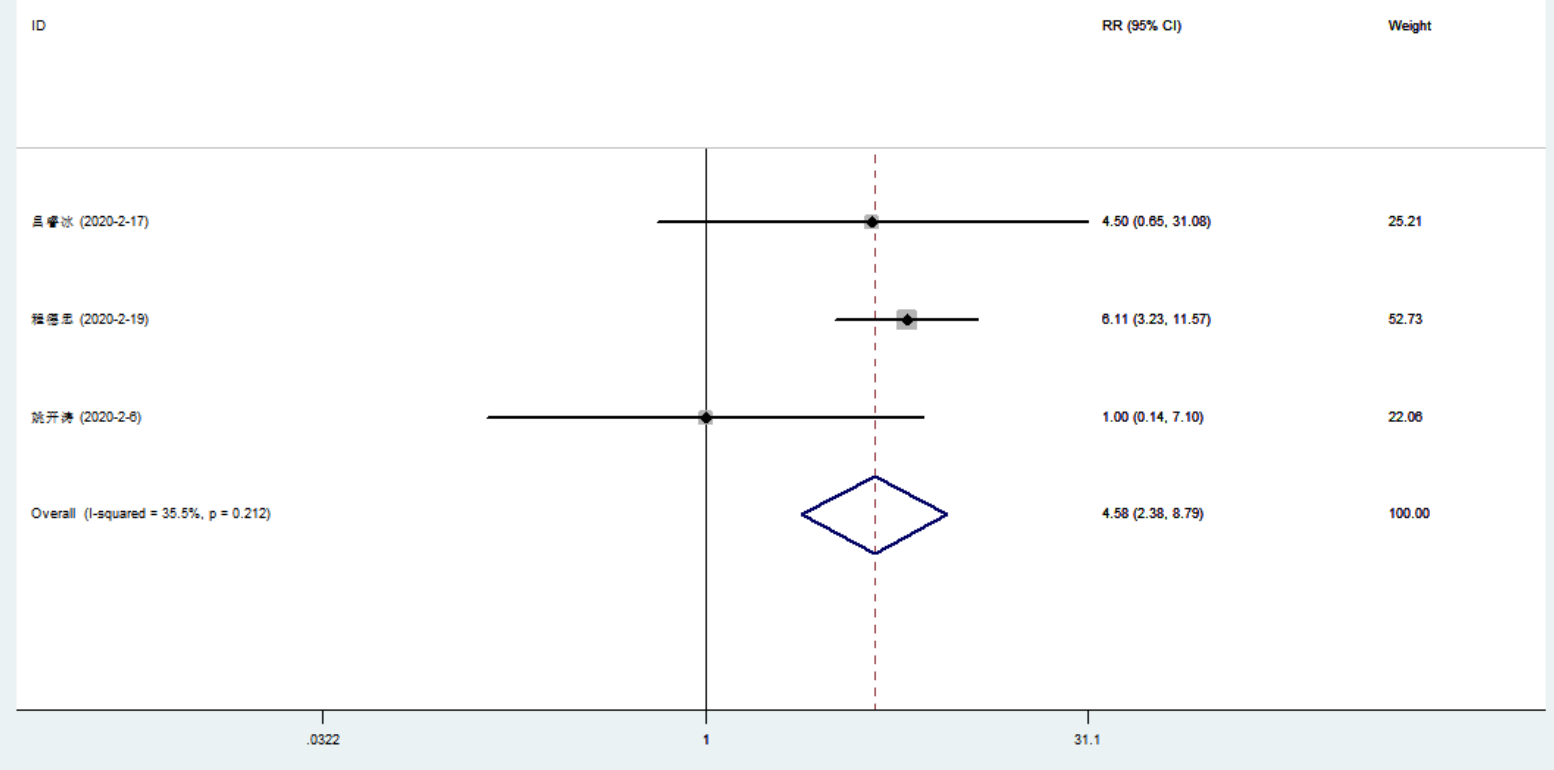

Figure 6. Meta-analysis forest plot of the disappearance of dyspnea between the combined group and the control group

Study

ID

RR (95\% CI)

Weight

三佂 $(2020-2-17)$

程嗦忠 (2020-2-19)

姚并潾 $(2020-2-8)$

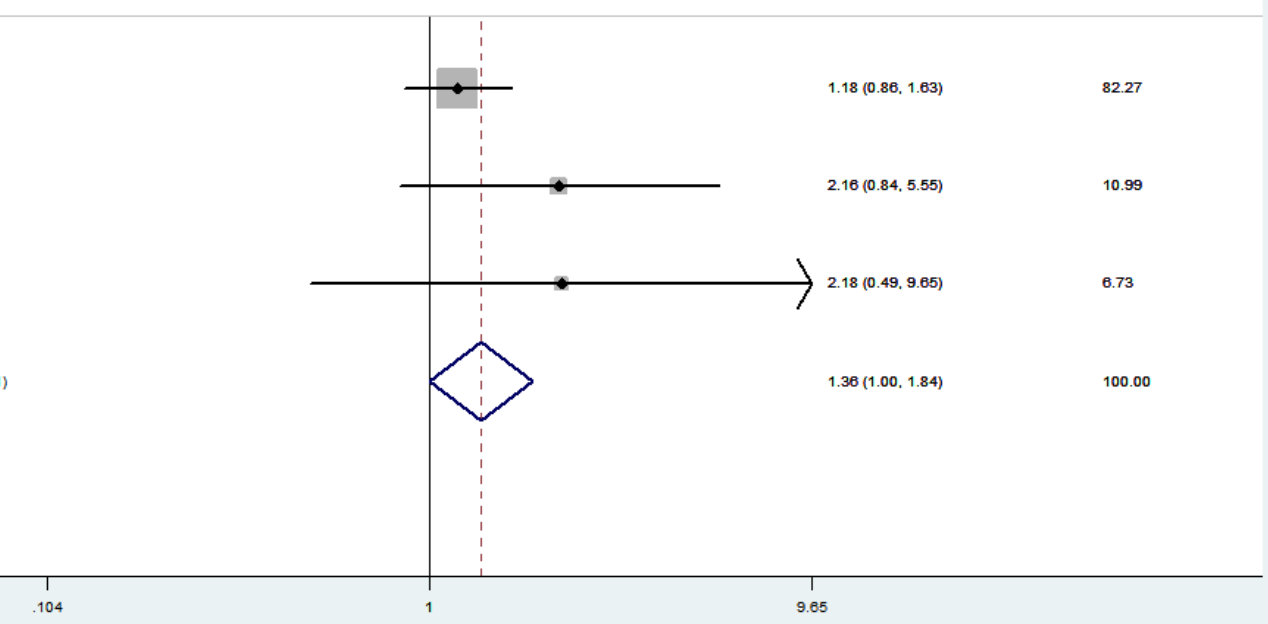

82.27

Overall (1-squared $=1.9 \%, p=0.361$ )

Figure 7. Meta-analysis forest plot of loss of appetite between the combined group and the control group 
Sensitivity analysis and publication bias analysis: There were no significant heterogeneity in the included studies, and the results of combining the two effect models were roughly the same. Begg's funnel plots and Egger's regression method were used to test the publication bias. The funnel plot shows two groups of basically symmetrical distributions, suggesting that the publication bias is small.

\section{Discussion}

Recently, Novel Coronavirus Pneumonia (NCP) has shown an outbreak trend, spreading in all provinces, cities and regions in China and even globally. It has been classified as an international public health emergency of major concern. The disease is mainly manifested by fever, fatigue, and dry cough. It can be seen that breathing is difficult and life-threatening. At present, there are no specific anti-viral treatment drugs and corresponding vaccines for NCP. The main clinical practice is symptomatic supportive treatment and comprehensive intervention. The severity of the disease and shortening the course have important clinical significance.

Lianhua Qingwen has been recommended by the State Administration of Traditional Chinese Medicine as a drug for the prevention and treatment of infectious diseases of the respiratory system. ERS-CoV [9] has a certain inhibitory effect. Lianhua Qingwen Capsules (particles) can inhibit viral infections through direct and indirect routes. The direct pathway mainly affects the function of the viral envelope, inhibits virus adsorption and penetration into cells, and inhibits viral transcription and replication processes. Indirect pathways include improving body immunity, improving immunoglobulin levels, regulating cytokine synthesis and release, and inhibiting oxidative stress damage caused by viruses, etc. [10]. In addition, Lianhua Qingwen Capsules (granules) also have significant clinical effects in antipyretic, cough and phlegm elimination, and antibacterial and anti-inflammatory effects [5]. According to the prediction of action targets, Lianhua Qingwen has 22 core targets for $2019-\mathrm{nCoV}$, mainly involving inflammatory mediators and mitogen-activated protein kinases [11]. Multiple clinical observations of Lianhua Qingwen combined with conventional treatment can increase the disappearance rate of major symptoms of NCP (fever, cough, fatigue) and other symptoms (chest tightness, dyspnea, and loss of appetite), and reduce the proportion of severe symptoms.

A total of 3 literatures were included in this meta-analysis, with 138 cases in the experimental group and 113 cases in the control group. The results showed that compared with conventional drugs, Lianhuaqingwen combined with conventional drugs increased the disappearance rate of major symptoms in patients with common NCP, and also improved secondary symptoms such as dyspnea and loss of appetite, indicating that the drug can help improve The clinical symptoms suggest that the drug has positive clinical application value for the treatment of NCP. This article compares the difference in the disappearance rate of chest tightness between Lianhuaqingwen combined with conventional medicine and conventional medicine in NCP patients. There is statistical heterogeneity in the included study groups. The clinical subjectivity of the disappearance of chest tightness is not ruled out. Indicators. In addition, chest tightness, as one of the secondary symptoms associated with the disease, has no significant effect on the clinical efficacy of the drug. This article compared the incidence of adverse reactions of Lianhua Qingwen combined with conventional drugs and conventional drugs on COVID-19, and found that during the treatment, no abnormalities related to Lianhua Qingwen granules were found in the treatment group. There was no statistical difference, suggesting that Huaqingwen capsule (granule) has good clinical application safety. The combined results of the application of the two effect models in this systematic evaluation are roughly the same. The publication bias is tested. The funnel plot shows that the two groups are basically symmetrically distributed, suggesting that the publication bias is small and statistically significant. In summary, through a systematic review of the literature, Lianhua Qingwen combined with conventional drugs can better alleviate the clinical symptoms of ordinary patients, has good safety, and has important clinical application value.

This study has the following deficiencies: (1) the number of included studies is small, and the study sites are in China, and it is difficult to perform subgroup analysis on other regions and races; (2) the diagnostic criteria used in the study are different, so the selectivity is not completely excluded Possibility of bias; (3) Most studies did not perform TCM syndrome classification for NCP patients and could not be analyzed according to TCM syndrome classification; (4) The quality of included literature is low, and high-quality multicenter large-sample randomized double-blind controlled trials are needed to verify; $\mathbb{Q}$ Although no publication bias was found through statistical methods, the possibility of publication bias was not completely ruled out.

\section{Fund project}

Project "Emergency Prevention and Control of New Coronavirus Infection" of the Science and Technology Department of Yunnan Province.

\section{References}

1. Huang C, Wang Y, Li X, Ren L, Zhao J, et al. (2020) Clinical features of patients infected with 2019 novel coronavirus in Wuhan, China. The Lancet 395: 497-506.

2. Wang D, Hu B, Hu C, Zhu F, Liu X, et al. (2020) Clinical characteristics of 138 hospitalized patients with 2019 novel coronavirus-infected pneumonia in Wuhan, China. Jama.

3. Li Liuyi, Wu Anhua (2020) Discussion on common confusions in the prevention and control of nosocomial infections of new coronaviruses. Chinese Journal of Infection Control 19: 1-4.

4. Dong L, Xia JW, Gong Y, Chen Z, Yang HH, et al. (2014) Effects of Lianhua Qingwen (LHQW) capsules on the induction of sputum inflammatory factors in patients with chronic obstructive pulmonary disease acute exacerbation (AECOPD). Fudan Journal (Medical Edition) 41: 243-247.

5. Lu Ruibing, Wang Wenju, Li Xin (2017) Clinical Observation on 63 Cases of Suspected Cases of New Coronavirus Pneumonia Treated by Lianhua Qingwen of Traditional Chinese Medicine. Journal of Traditional Chinese Medicine pp: 1-5.

6. Yao K, Liu MY, Li X, Huang JH, Cai HB (2020) Retrospective clinical analysis on treatment of novel coronavirus-infected pneumonia with traditional Chinese medicine Lianhua Qingwen. Chin J Exp Tradit Med Form 2020: 1-7.

7. Cheng Dezhong, Li Yi (2020) Clinical analysis and typical case report of Lianhua Qingwen Granules in treating 54 patients with new type of coronavirus pneumonia. World Journal of Traditional Chinese Medicine pp: 1-5.

8. Zhu Shunya, Li Xiaozheng, Wei Yunling (2003) Preliminary study on the in vitro inhibitory effect of three traditional Chinese medicine prescriptions on SARSassociated coronavirus [D].

9. Guan Wenda, Du Qiuling, Jiang Haiming (2020) Comparison of inhibitory effects of arbidol and Lianhuaqingwen Capsules on Middle East respiratory syndrome coronavirus in vitro and in vivo. Guangdong Medical Journal 039: 3454-3458.

10. Lin Quan, Miao Changxin, Wei Xuhua (2020) Efficacy of Lianhua Qingwen Granules combined with azithromycin in sequential treatment of children with Mycoplasma pneumoniae pneumonia combined with atelectasis and its effect on T lymphocyte subsets and inflammatory factors [J]. Modern Journal of Integrated Traditional Chinese and Western Medicine 28: 47-52.

11. Wang Lin, Yang Zhihua, Zhang Haoran (2020) Network Pharmacological Study and Preliminary Evidence of Lianhua Qingwen in the Treatment of New Coronavirus (2019-nCoV) Pneumonia. Chinese Medicinal Materials pp: 1-8.

Copyright: (C2020 Ren Q. This is an open-access article distributed under the terms of the Creative Commons Attribution License, which permits unrestricted use, distribution, and reproduction in any medium, provided the original author and source are credited. 